\title{
Desarrollo de curvas de preferencia de microhábitat para Leuciscus pyrenaicus y Barbus bocagei por buceo en el río Jarama (Cuenca del Tajo).
}

\author{
F. Martínez Capel \& D. García de Jalón Lastra. \\ Laboratorio de Hidrobiología. E.T.S. de Ingenieros de Montes. Ramiro de Maeztu s/n. MADRID 28040.
}

\begin{abstract}
RESUMEN
La aplicación de la Metodologia IFIM (Instream Flow Incremental Methodology) en la Península Ibérica requiere el desarrollo previo de curvas apropiadas para los ciprinídeos endémicos. En el presente estudio, hicimos un muestreo en un tramo en el Río Jarama (cuenca del Tajo) por medio de observación subacuática, de modo que fuesen establecidas las preferencias de microhabitat de Barbus bocagei (93 Ind.) y Leuciscus pyrenaicus (543 Ind.). Las curvas para los barbos muestran preferencias máximas para velocidades de $5 \mathrm{~cm} / \mathrm{s}$ (adultos, AD), $20 \mathrm{~cm} / \mathrm{s}$ (juveniles, JV) y $25 \mathrm{~cm} / \mathrm{s}$ (alevines, AL). Los valores máximos de profundidad fueron $121 \mathrm{~cm}(\mathrm{AD}), 56 \mathrm{~cm}(\mathrm{JV})$ y $51 \mathrm{~cm}$ (alevines). Respecto al substrato, los adultos prefirieron sobre todo fondos rocosos, los juveniles prefirieron arena y los alevines cantos rodados. Las curvas de preferencia para el cacho mostraron el máximo a $30 \mathrm{~cm} / \mathrm{s}(A D), 40 \mathrm{~cm} / \mathrm{s}(J V)$ y $15 \mathrm{~cm} / \mathrm{s}(A L)$. Los valores máximos de profundidad fueron a $126 \mathrm{~cm}$ $(A D), 46 \mathrm{~cm}(J V)$ y $121 \mathrm{~cm}(A L)$, y la grava fue el substrato más apropiado para los tres estados de vida.
\end{abstract}

Palabras clave: IFIM, microhábitat, preferencias, Barbus bocagei, Leuciscus pyrenaicus.

\begin{abstract}
The application of the Instream Flow Incremental Methodology in the Iberian Peninsula first requires the development of suitability curves for the endemic cyprinids. In the present study, we sampled a reach in the River Jarama (basin of River Tagus) by underwater observation, in order to establish the microhabitat preferences of Barbus bocagei (93 ind.) and Leuciscus pyrenaicus (543 ind.). The curves for barbel showed preference of this species for water velocities of $5 \mathrm{~cm} \mathrm{~s}^{-1}$ for adults (AD), 20 $\mathrm{cm} \mathrm{s}^{-1}$ for juveniles (JV), and $25 \mathrm{~cm} \mathrm{~s}^{-1}$ for young of the year (YOY). Preferred depths were $121 \mathrm{~cm}(\mathrm{AD}), 56 \mathrm{~cm}(\mathrm{JV})$ and 51 $\mathrm{cm}$ (YOY). As regards substrate, adults preferred bedrock overall, juveniles preferred sand, and young of the year preferred cobbles. For Spanish chub, preferred water velocities were $30 \mathrm{~cm} \mathrm{~s}^{-1}(\mathrm{AD}), 40 \mathrm{~cm} \mathrm{~s}^{-1}(\mathrm{JV})$ and $15 \mathrm{~cm} \mathrm{~s}^{-1}$ (YOY). For depth, identified preferences were $126 \mathrm{~cm}(\mathrm{AD}), 46 \mathrm{~cm}(\mathrm{JV})$ and $121 \mathrm{~cm}(\mathrm{YOY})$, and gravel was the preferred substrate type for the three life stages.
\end{abstract}

Keywords: IFIM, microhabitat, suitability, Barbus bocagei, Leuciscus pyrenaicus.

\section{INTRODUCCIÓN}

En los últimos años, se han realizado en España un gran número de estudios para el cálculo de regímenes de caudales ecológicos, siendo muy frecuente el uso de la simulación del hábitat físico propuesta dentro de la metodología IFIM (Instream Flow Incremental Methodology;
Bovee, 1982). Para esta simulación, resulta necesario el desarrollo previo de curvas de preferencia para algunas especies piscícolas, es decir, la modelización de sus requerimientos vitales en términos de variables hidráulicas del hábitat físico. Actualmente, se necesita un mayor estudio de curvas de preferencia experimentales, sobre todo cuando se trata de especies endémicas de la 
Península Ibérica, ya que carecemos de datos procedentes de otros países. El uso del microhábitat para algunas especies de macroinvertebrados, salmónidos y ciprínidos ha sido estudiado experimentalmente con anterioridad (Grossman et al., 1987a, b; Grossman \& Freeman, 1987; Brotons, 1988; Grossman \& Sostoa, 1994a, b; Mayo et al., 1995). En relación con las especies endémicas de ciprínidos, solo contamos con las curvas de probabilidad de uso realizadas por Costa et al. (1988) y las curvas de preferencia propuestas por García de Jalón et al. (1997).

El objetivo principal de este trabajo era obtener curvas de preferencia (categoría III; Bovee, 1986) para los ciprínidos endémicos más representativos en un tramo del río Jarama, y que resultaron ser el barbo común (Barbus bocagei) y el cacho (Leuciscus pyrenaicus). Se trataba de obtener unas curvas validas "in situ" para el río Jarama, que pudiesen implementarse en un modelo de simulación del hábitat físico. Este estudio forma parte de una tesis doctoral sobre las preferencias de microhábitat del barbo común, la boga y el cacho, donde el método escogido es la observación directa bajo el agua.

Las curvas de preferencia nos muestran la conveniencia de los valores de una determinada variable del microhábitat, dentro del rango estudiado en cada zona de estudio. Estas curvas se basan en el estudio de la probabilidad de uso ponderado según el hábitat disponible, en cada estación de muestreo. De este modo se intenta corregir el efecto producido por la distinta disponibilidad de microhábitats, que influye sobre el comportamiento de los individuos en cada lugar. Nos basamos en el concepto de selección como el uso de un recurso en una proporción superior de aquella en la que el recurso se encuentra disponible en la estación (Manly et al., 1993). A partir de esta idea, empleamos un índice de selección que consiste en el cociente entre la proporción de uso para un rango de la variable estudiada y la proporción en que este rango de la variable se encuentra disponible. Este índice es el más sencillo y comúnmente utilizado para este tipo de estudios (Bovee, 1998).

\section{ÁREA DE ESTUDIO}

La estación se situó en un tramo del río Jarama, afluente por la derecha del río Tajo. La estación se sitúa aguas arriba de su convergencia con el río Lozoya, en el paraje conocido como "Casas de los Quintanares" (Guadalajara). Se trata de un tramo de orden 4 , con un caudal medio anual de $5 \mathrm{~m}^{3} / \mathrm{s}$ y que se encuentra regulado por la presa del Vado, a $19 \mathrm{~km}$ aguas arriba de este punto. Hay que resaltar que el río mantiene caudal todo el año, sin llegar a secarse. En este sentido se calculó también la media del caudal mínimo anual, $0.25 \mathrm{~m}^{3} / \mathrm{s}$, extraído de los datos de aforos de la serie 1974-1986 (tras la construcción de la presa del Vado), de donde procede también el valor del módulo anual.

La longitud del tramo era de $241 \mathrm{~m}$, y su anchura media $6.81 \mathrm{~m}$. Se encontraba en una zona de media montaña, con una pendiente media del agua de $1.67 \times 10^{-3}$. Se tipificaron los mesohábitats, encontrándose una sucesión (hacia aguas abajo) de poza, corriente, poza, corriente y poza. La relación rápidos/lentos era de 0.18 , y la distancia relativa entre rápidos 15 $\mathrm{m}$. La vegetación de ribera se encontraba en buenas condiciones y era abundante, predominando el aliso en todas las orillas, seguido del sauce y el chopo blanco. La temperatura media del agua fue de $18^{\circ} \mathrm{C}$. En cuanto a la comunidad piscícola, solo se pretendía obtener datos cualitativos. Fruto de la observación directa bajo el agua y de varios muestreos de pesca eléctrica, se determinó que la composición de la comunidad piscícola (en orden de importancia) era: Cacho, barbo, gobio, lucio y pez sol.

\section{METODOLOGÍA}

En primer lugar hay que decir que para caracterizar cada microhábitat hemos empleado las variables de velocidad, profundidad y sustrato, contando también con las variables de presencia de refugio disponible para el pez y distancia a la orilla (de las cuales se hace un análisis diferente). A pesar de que otros autores han estudiado 
numerosas variables físicas (ver Jowett, 1990) en la mayoría de los casos todas dependen de los 4 primeros factores (p.e.: Wesche et al. 1987; Jowett, 1990), exceptuando las variables relacionadas con la temperatura.

El muestreo subacuático del uso del hábitat se llevó a cabo entre el 17 de Junio y el 10 de Julio de 1997. La persona que bucea se dirige corriente arriba comenzando por el punto inferior de la estación y observa a cierta distancia la situación de los individuos, ya sean aislados o en grupos, procurando no molestarles. Si se observa que el pez está siendo perturbado en su comportamiento, no se realiza el registro. El buceador lleva un conjunto de marcadores preparados para colocarlos donde se encuentra cada pez o conjunto observado. Primero, al observar a cierta distancia, se apunta (en una tablilla subacuática) los siguientes datos: Número de registro (chapa numerada adjunta al marcador), especie, estado de desarrollo (alevín, juvenil o adulto), altura focal estimada "de visu", elementos de refugio cercanos disponibles para el pez, número de individuos situados en dicho microhábitat y observaciones que estime oportunas (generalmente sobre su comportamiento). Entonces, se coloca sobre el lecho el marcador, que está hecho de una pequeña pieza de acero numerada y con un lazo rojo atado.

El criterio adoptado para separar las etapas de desarrollo de las dos especies estudiadas (barbo y cacho) ha sido principalmente la estimación de la longitud de los individuos, así como otros criterios adicionales, como la presencia de tubérculos nupciales claramente visibles en el caso de muchos barbos adultos. Las clases de longitud tomadas para el barbo han sido de 0 a $7 \mathrm{~cm}$ (alevín), de 7 hasta $25 \mathrm{~cm}$ (juvenil) y mayor de $25 \mathrm{~cm}$ (adulto). Para el cacho han sido: 0-5 cm (alevín), de 6 a $10 \mathrm{~cm}$ (juvenil) y de $10 \mathrm{~cm}$ en adelante (adulto).

Una vez recorrida toda la estación, dos muestreadores miden las variables del microhábitat en cada uno de estos puntos: velocidad media de la columna de agua, velocidad focal, profundidad, altura focal, tipo de sustrato y distancia del punto a la orilla. Se anotan también los elementos de refugio cercanos y la existencia de sombra.
Para la medición de las variables del microhábitat en cada punto se ha seguido la metodología que se expone a continuación.

- Profundidad y altura focal: La altura focal es la distancia sobre el lecho a la que se encuentra situado el pez, y a la cual se mide posteriormente la velocidad focal. El buceador la estima "de visu" en centímetros o como porcentaje del calado en el punto y, posteriormente (cuando se vuelve a pie), se mide en $\mathrm{cm}$ la longitud a que corresponde esta distancia, así como el calado total en el punto en $\mathrm{cm}$. Esto se hace con una vara graduada y precisión de $1 \mathrm{~cm}$, al igual que las medidas de calado que se hacen en los transectos y para conocer la distancia del punto a la orilla.

- Velocidad y velocidad focal: En cada uno de los puntos escogidos por el pez se miden la velocidad focal y la media, mientras que en los transectos se mide solamente la velocidad media. Para ello se utilizó un aparato Valeport modelo BFM 002 (de precisión $0.01 \mathrm{~m} / \mathrm{s}$ ) y la velocidad media en la columna de agua se calculó del modo siguiente. Cuando el calado es mayor de $70 \mathrm{~cm}$ se midió la velocidad a dos profundidades, iguales al $80 \%$ y $20 \%$ del calado; el promedio de las dos medidas es la velocidad media de la columna de agua. Donde el calado es menor de $70 \mathrm{~cm}$, se realizó una sola medición, a una profundidad igual al $60 \%$ del calado en ese punto.

- Tipos de Sustrato: Se ha estudiado según la clasificación granulométrica de la American Geophysical Union traducida del siguiente modo: Roca madre (Roca continua), Bloques (>1024 $\mathrm{mm})$, Bolos (256-1024 mm), Cantos rodados (64$256 \mathrm{~mm})$, Gravas (8-64 mm), Gravillas (2-8 $\mathrm{mm})$, Arenas $(62 \mathrm{~mm}-2 \mathrm{~mm})$ y Limo $(<62 \mathrm{~mm})$. Para el uso del microhábitat se registra el sustrato dominante en un determinado radio alrededor del pez (Hampton \& Aceituno, 1988), aproximadamente $15 \mathrm{~cm}$. En los transectos de muestreo se toma el tipo de sustrato dominante en cada celda.

- Refugio: Se toma nota de la presencia de elementos de cobertura (hasta una distancia máxima de $1 \mathrm{~m}$ ). En el presente trabajo solo se ha analizado la frecuencia de peces asociados a dos tipos distintos de refugio, el que es suministrado por la vegetación existente dentro del 

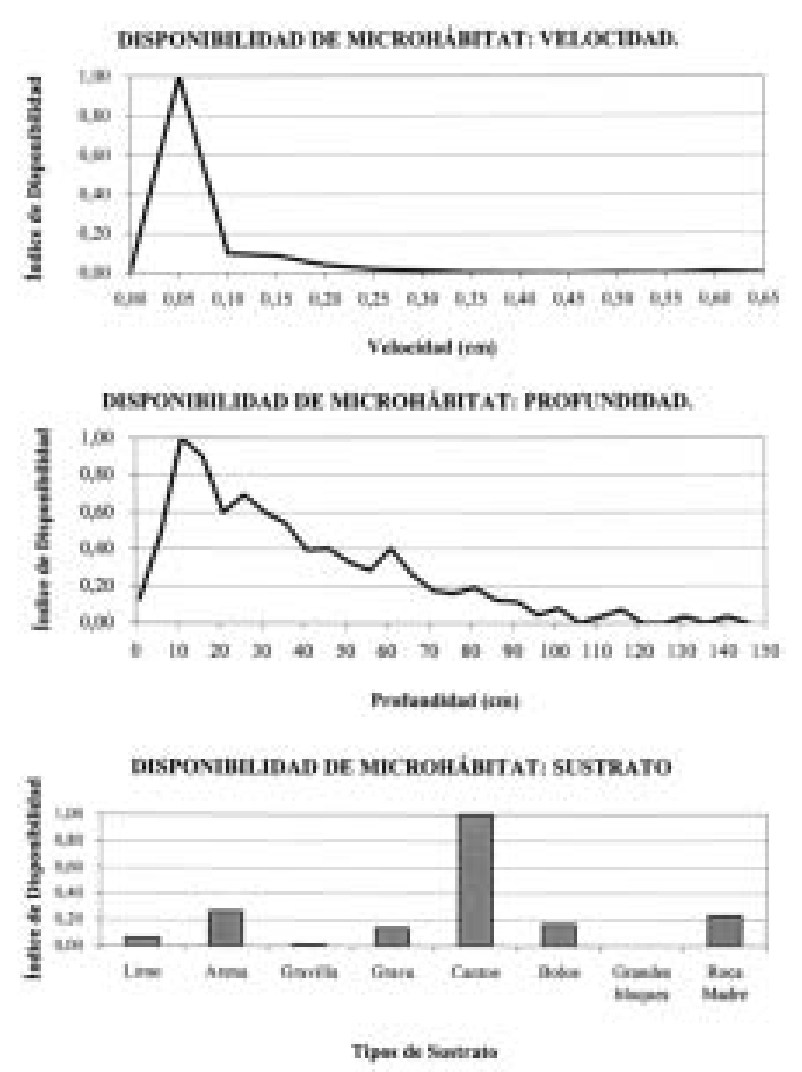

Figura 1. Curvas de disponibilidad de microhábitat para velocidad, profundidad y sustrato. Microhabitat availability curves for water velocity, depth and substrate.

cauce o en sus orillas (vegetación macrofítica de especies sumergidas o emergentes, raíces sumergidas, ramillas, ramas o troncos sumergidos -vivos o muertos- y sombra), y el que aporta el sustrato del lecho o las orillas sin relación directa con seres vivos (cornisas sumergidas o semisumergidas y cuevas u oquedades entre piedras de distintos tamaños).

Una vez que se han registrado las condiciones de los puntos escogidos por los individuos, se pasa a muestrear el hábitat disponible del tramo. Para ello se realizaron 23 transectos, situados de modo que representaran adecuadamente la heterogeneidad longitudinal del tramo (zonas de poza profunda, tablas, rápidos, etc.) y de modo que los puntos de medición en cada uno (13 como media por transecto) reflejaran la topografía del lecho y las con- diciones hidráulicas de la sección tomada (de acuerdo con la metodología de simulación física del hábitat de IFIM). A partir de estos datos podemos estudiar la presencia de zonas con distintas profundidades, velocidades, tipos de sustrato y disponibilidad de refugio que existen en el tramo de estudio. Esto es muy importante, ya que la preferencia del pez está en función del espectro ecológico de la especie y del hábitat disponible (Grossman et al., 1987a,b; Heggenes et al., 1991).

En primer lugar se asignó una representatividad a cada transecto, que es el porcentaje de la longitud de río representada por el transecto respecto a la longitud total del tramo (redondeando a un número entero). Para un transecto ' $i$ ' lo llamaríamos $\mathrm{RT}_{i}$, A continuación se calcula una frecuencia $\mathrm{Fj}$ para cada clase ' $j$ ' en que se ha dividido todo el rango de valores del parámetro (en el caso de velocidad o profundidad) o cada tipo de sustrato. El número de medidas en cada transecto se multiplica por la representatividad del mismo, y se suman las frecuencias de cada clase para todos los transectos, obteniendo la suma total de frecuencias para cada clase ' $j$ '. Con estas frecuencias se calcula un "Indice de Disponibilidad" para cada intervalo $\mathrm{j}$ del parámetro. Este índice, Idj, consiste en el cociente entre la frecuencia ponderada de la clase Fj y la suma de las frecuencias ponderadas de todas las clases, es decir:

$$
\operatorname{Idj}=\frac{F j}{\sum_{j} F j}
$$

$\mathrm{Su}$ representación gráfica, normalizando la curva entre 0 y 1 , es la que se ha llamado curva de disponibilidad de microhábitat de cada variable.

Por otra parte, refiriéndonos al uso del microhábitat por parte de los peces, utilizamos un Indice de Uso para cada clase $\mathrm{j}$ del parámetro, definido como:

$$
\mathrm{Iuj}=\frac{\mathrm{N}^{\mathrm{o}} \text { individuos asociados a la clase } \mathrm{j}}{\mathrm{N}^{\mathrm{o}} \text { total de individuos }}
$$


La representación gráfica de este índice, normalizado a 1, es la que se ha llamado curva de uso de microhábitat de cada variable, para una especie y etapa de desarrollo determinada.

Para obtener una curva de preferencia de categoría III se pondera el uso del hábitat con el hábitat disponible en el tramo. Esto se hace dividien-

\section{Barbo adulto}

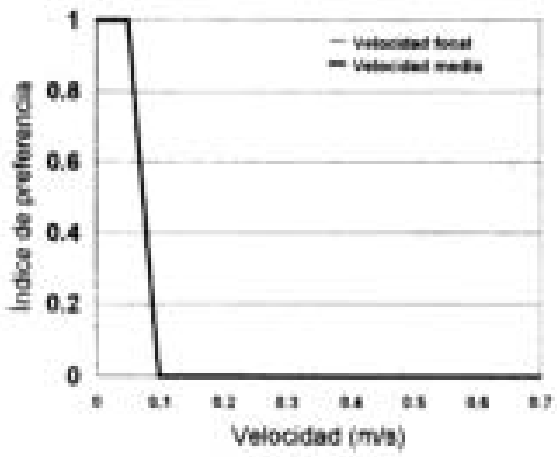

Barbo juvenil

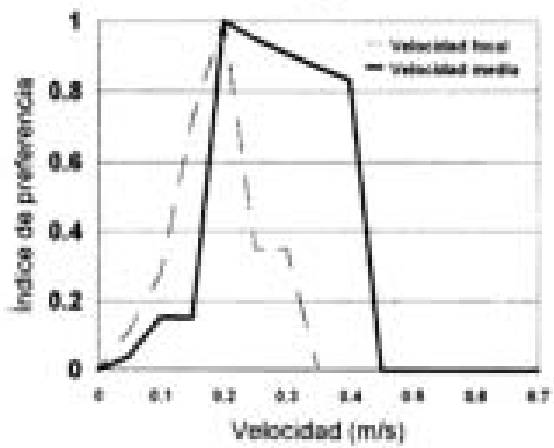

Barbo alevin

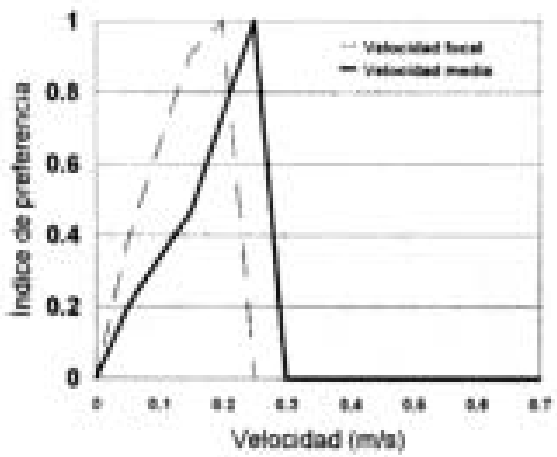

do la primera serie de datos (uso del hábitat) por la segunda (hábitat disponible), obteniendo así un índice de selección, es decir:

$$
\mathrm{Cj}=\frac{\text { Iuj }}{\text { Idj }}
$$

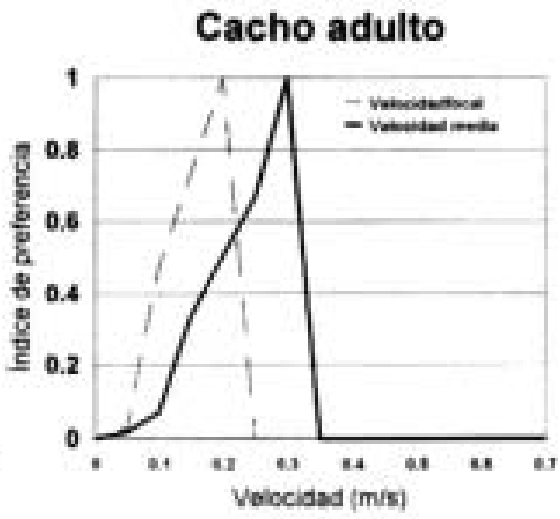

Cacho juvenil

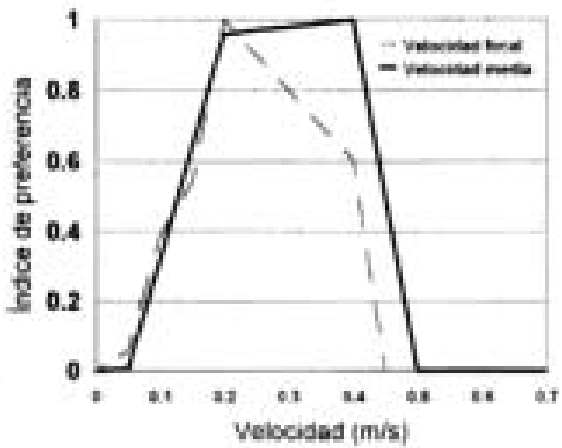

Cacho alevin

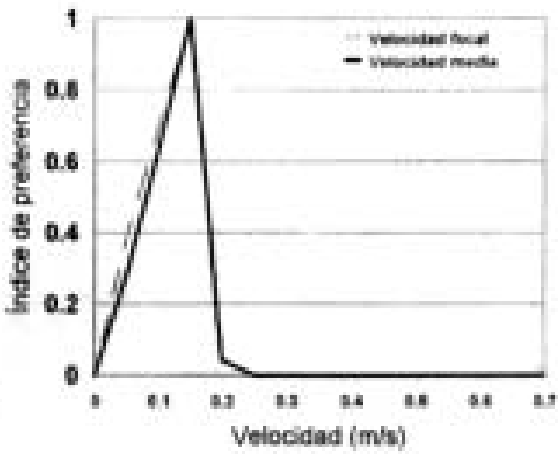

Figura 2. Curvas de preferencia de velocidad media y focal. Suitability curves for focal and mean water velocity. 
Por último, los índices obtenidos se representan por una curva normalizada entre 0 y 1. Obtenemos así la curva de preferencia buscada para el parámetro, especie y etapa de desarrollo de que se trate. Las curvas resultantes han sido suavizadas para los parámetros continuos, de modo que se presentan las curvas envolventes. El parámetro sustrato se presenta en forma de diagrama de barras.

\section{RESULTADOS}

Al estudiar el microhábitat disponible, se realizaron las curvas de disponibilidad para las variables de velocidad, profundidad y sustrato, que se muestran en la figura 1. En cuanto a la velocidad, predominaban las zonas lentas, midiéndose un gran número de puntos $(77.8 \%)$ con velocidad menor de $0,05 \mathrm{~m} / \mathrm{s}$, y con un máximo de $0.703 \mathrm{~m} / \mathrm{s}$. El análisis de las profundidades reveló que un gran porcentaje del hábitat $(75 \%)$ presentaba profundidades iguales o menores a $50 \mathrm{~cm}$, habiéndose encontrado una zona de poza donde se alcanzó el máximo de 1.4 $\mathrm{m}$ de profundidad. Por último, para el sustrato se observa que los cantos rodados representan un porcentaje muy importante $(53.6 \%)$ y la arena aparece la segunda en abundancia (14\%).

En el muestreo subacuático del uso del hábitat se realizaron en total 102 registros, registrando la preferencia mostrada por 636 individuos.

Tabla 1. Valores medios de porcentaje de altura focal, con su desviación estándar, para cada especie y etapa de desarrollo. BB: Barbus bocagei; LP: Leuciscus pyrenaicus. Average and standard deviation of the percentage of focal height, for all species and life stages. BB: Barbus bocage ; LP: Leuciscus pyrenaicus.

\begin{tabular}{lcc}
\hline \% Alt. Focal & Media & Desv. Estándar \\
\hline BB adulto & 15 & 3 \\
BB juvenil & 20 & 12 \\
BB alevin & 15 & 5 \\
LP adulto & 14 & 6 \\
LP juvenil & 17 & 11 \\
LP alevin & 21 & 17 \\
\hline
\end{tabular}

Los cachos (Leuciscus pyrenaicus) contaban 543 (35 adultos, 269 juveniles y 239 alevines) y los barbos (Barbus bocagei) 93, de los cuales 6 eran adultos, 62 juveniles y 25 alevines. Ninguno de ellos mostraba un comportamiento migratorio ni reproductivo y no se detectó ningún nido. Las curvas de preferencia (para velocidad, profundidad y sustrato) se calcularon para cada especie y etapa de desarrollo

\section{Velocidad media y velocidad focal (Fig. 2).}

En el caso del barbo, los juveniles y alevines mostraron máximos de velocidad focal en la clase $0.16-0.20 \mathrm{~m} / \mathrm{s}$, mientras que para los adultos (en las zonas más lentas del tramo) el óptimo es menor de $0.05 \mathrm{~m} / \mathrm{s}$. Los cachos juveniles y adultos presentan las mismas preferencias que barbos juveniles y alevines, mientras que los alevines prefieren $0.11-0.15 \mathrm{~m} / \mathrm{s}$. En general la velocidad media aparece ligeramente mayor a la focal preferida, lo cual responde a que en general los individuos se encontraban muy cerca del lecho.

\section{Profundidad total y altura focal (Fig. 3).}

Tanto cachos como barbos han sido encontrados cerca del sustrato, y las curvas de altura focal reflejan un máximo en 2-6 cm, decreciendo después rápidamente. En general el rango observado para los barbos ha sido menor que para los cachos, aunque los barbos adultos han sido la excepción con un óptimo de 12-16 cm. En cuanto a la profundidad, los adultos son los que presentan los óptimos más altos: de $117 \mathrm{~cm}$ en adelante para el barbo y de $122 \mathrm{~cm}$ en adelante para el cacho. Los juveniles prefieren profundidades menores, con un máximo en $52-56 \mathrm{~cm}$ para el barbo y en $42-46 \mathrm{~cm}$ para el cacho. Por último los alevines tienen preferencias muy distintas, con un pico en los $47-51 \mathrm{~cm}$ para el barbo y constantemente creciente en el cacho con el máximo en los 117-121 cm.

Se definió también el parámetro "porcentaje de altura focal" como el tanto por ciento de la altura focal respecto de la profundidad total en cada punto. Se calcularon los valores medios para cada especie y etapa de desarrollo obteniéndose valores entre un 15 y un $20 \%$ para el barbo -BB- y 


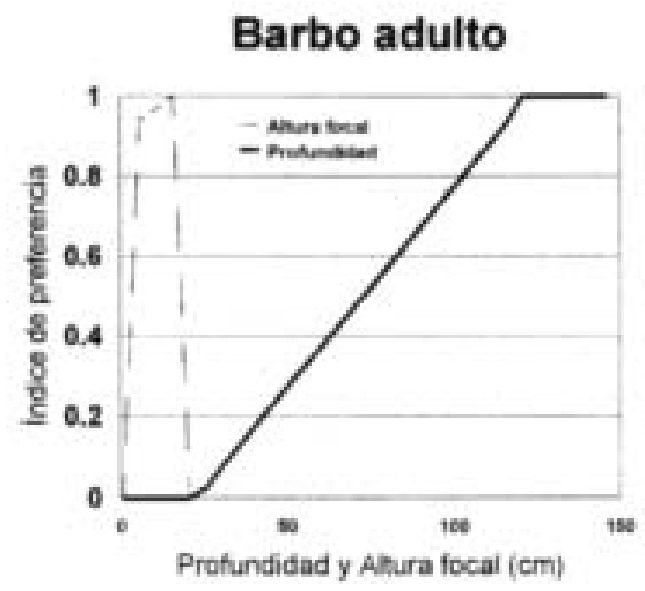

Barbo juvenil

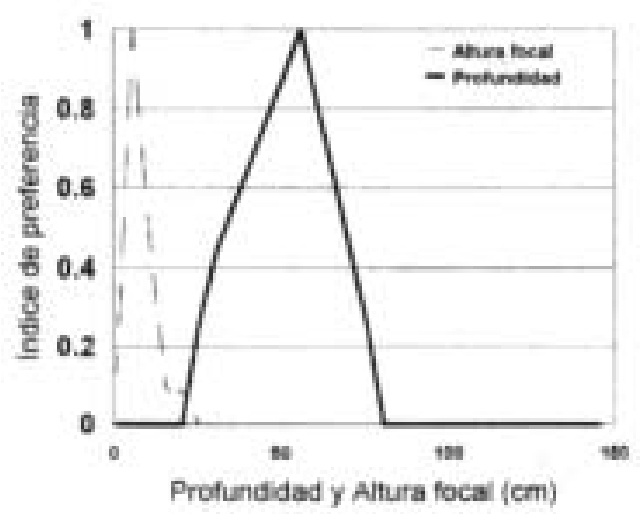

Barbo alevin

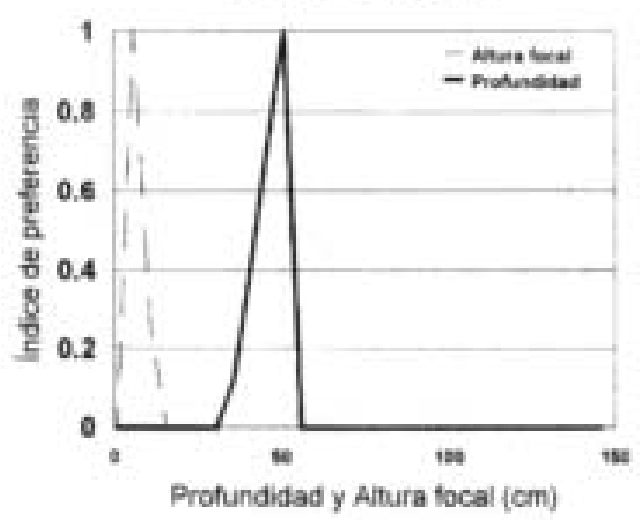

Cacho adulto

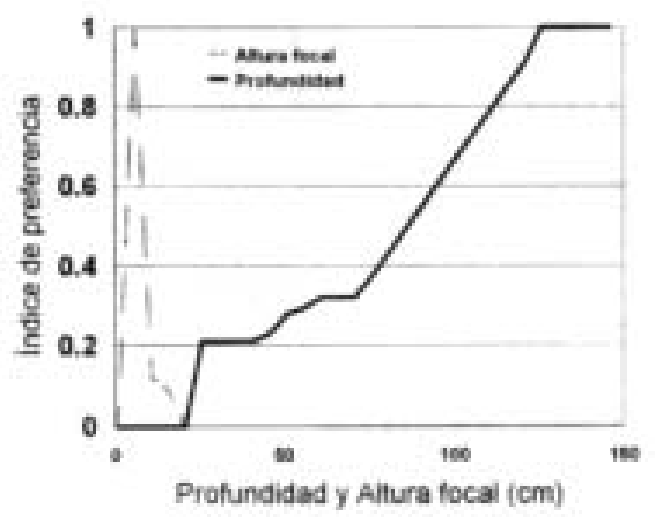

Cacho juvenil

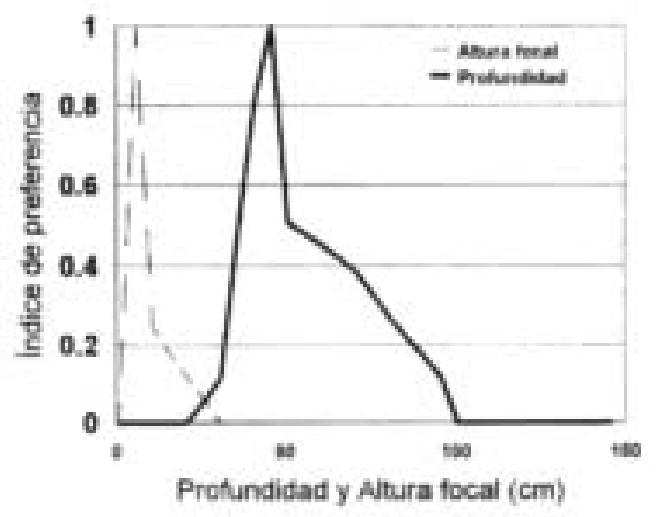

Cacho alevin

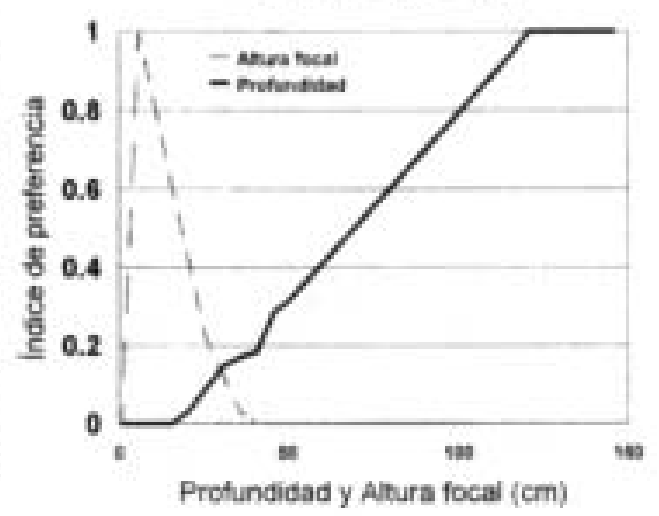


Tabla 2. Valores medios de distancia a la orilla, con su desviación estándar, para cada especie y etapa de desarrollo. BB: Barbus bocagei; LP: Leuciscus pyrenaicus. Average and standard deviation of distance to shore, for all species and life stages. BB: Barbus bocagei; LP: Leuciscus pyrenaicus.

\begin{tabular}{llc}
\hline Dist. Orilla & Media & Desv. Estándar \\
\hline BB adulto & 2 & 0 \\
BB juvenil & 1.5 & 1 \\
BB alevin & 0.7 & 1 \\
LP adulto & 2 & 1 \\
LP juvenil & 2 & 1 \\
LP alevin & 2 & 2 \\
\hline
\end{tabular}

del 14 al $21 \%$ para el cacho - LP - (resultados que aparecen en la tabla 1 , junto a sus desviaciones estándar). Estos resultados no han mostrado diferencias significativas entre especies o etapas.

\section{Sustrato (Fig. 4).}

Los barbos adultos presentan una preferencia máxima por la roca madre seguida de la grava (8$64 \mathrm{~mm}$ ), mientras que el cacho adulto prefería claramente la grava. Los barbos juveniles tienen su óptimo en la arena, decreciendo la preferencia al aumentar la granulometría de las partículas. Los cachos juveniles en cambio prefieren sobre todo la grava y en segundo lugar la gravilla (2-8 $\mathrm{mm})$. Por último, los alevines de barbo prefieren los cantos rodados $(64-256 \mathrm{~mm})$ y en segundo lugar las gravas, justamente al contrario que los alevines de cacho.

En cuanto a la distancia a la orilla, se calculó la media y desviación estándar, para cada especie y etapa de desarrollo (ver Tabla 2), comparándose además con la distancia media a la orilla de los puntos con refugio disponible (obtenido del muestreo por transectos) y con la anchura media del cauce $(6.8 \mathrm{~m})$. La distancia media en que se situaban los barbos fue de 1,3 m (siendo creciente con la edad) y $2 \mathrm{~m}$ para los cachos, comparado con la distancia media de los puntos de refugio que fue de 1.2 metros. Podemos decir que la mayoría del refugio se encuentra asociado a las orillas $(\mathrm{d}=1.2 \mathrm{~m})$, pero la situación de los peces en el cauce no se puede correlacionar de un modo claro con este parámetro. El barbo presenta una frecuencia media del $54 \%$ junto a elementos de refugio, al tiempo que su distancia media a la orilla se acerca mucho a la media del refugio disponible (1.2 m). En cambio los cachos son un $29 \%$, lo que concuerda con las diferencias entre su situación en el cauce y la media de los elementos de refugio disponibles.

En nuestro tramo de estudio, los barbos alcanzaban los $460 \mathrm{~mm}$, mientras que el cacho es una especie de menor talla (longitud máxima de 109 $\mathrm{mm}$ ), lo cual podría ser una razón clara de que precisen menos refugio por poder esconderse de la vista de un predador más fácilmente. Los porcentajes de frecuencias del refugio respecto al número total en cada especie y etapa de desarrollo, separados según esté un carácter relacionado con el sustrato o con la vegetación, se muestran en la figura 5. Cabe anotar que los elementos de refugio utilizados estaban relacionados en su mayoría con la presencia de vegetación en el río.

\section{DISCUSIÓN}

En cuanto a los barbos adultos, hemos coincidido con algunas de las observaciones de Grossman \& Sostoa (1994 a, b), según las cuales los individuos mayores se sitúan en las aguas más profundas, aunque en nuestra estación estaban asociados a las velocidades más bajas, sustratos de roca madre y refugio cercano. En el caso de los barbos consideramos que su elección está ligada a las zonas más profundas de pozas con el refugio que ofrecen las cuevas de la orilla, por lo cual la preferencia se mantendría máxima en el caso de que las profundidades del tramo fuesen mayores de $145 \mathrm{~cm}, \mathrm{y}$ de este modo lo hemos reflejado en su curva de preferencia. Aunque los cachos no necesitan tanta profundidad para refugiarse pensamos que por las mismas razones el índice de preferencia también se mantiene igual a 1 a partir de los $122 \mathrm{~cm}$ en adelante.

Los barbos juveniles seleccionaron velocidades parecidas a las de los cachos juveniles, encontrándose generalmente juntos en los cardú- 
Barbo adulto

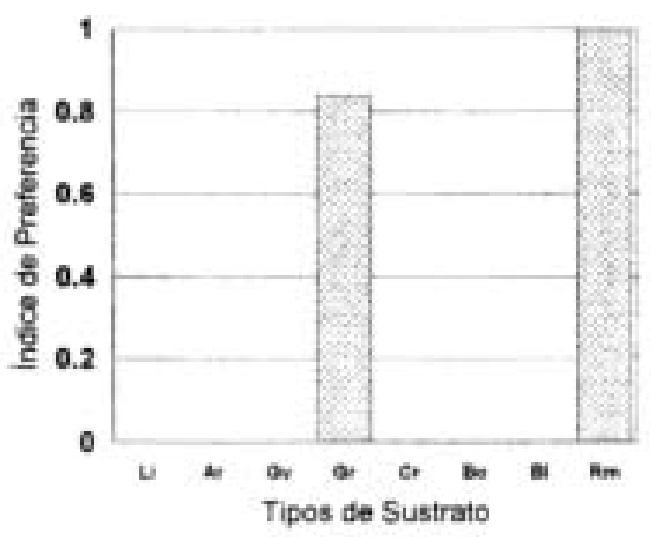

Barbo juvenil

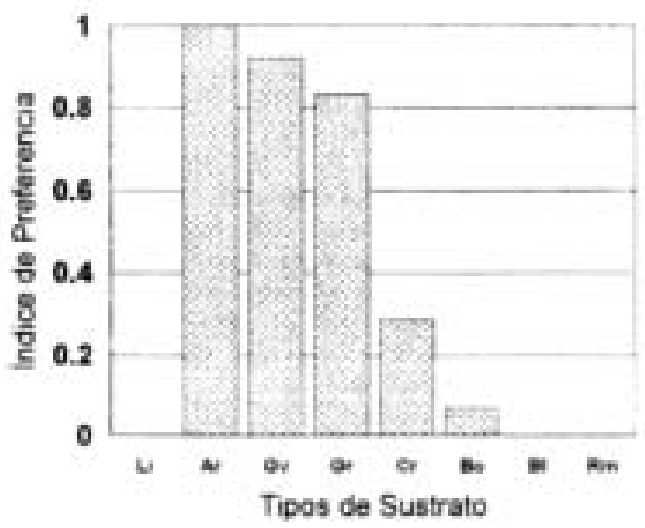

Barbo alevin

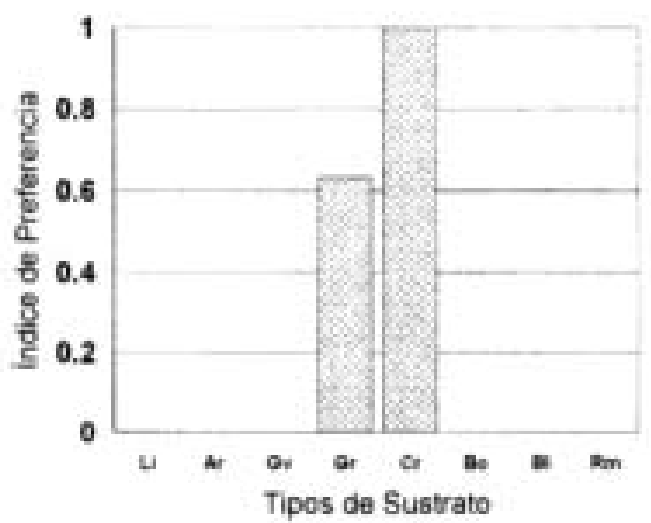

Cacho adulto

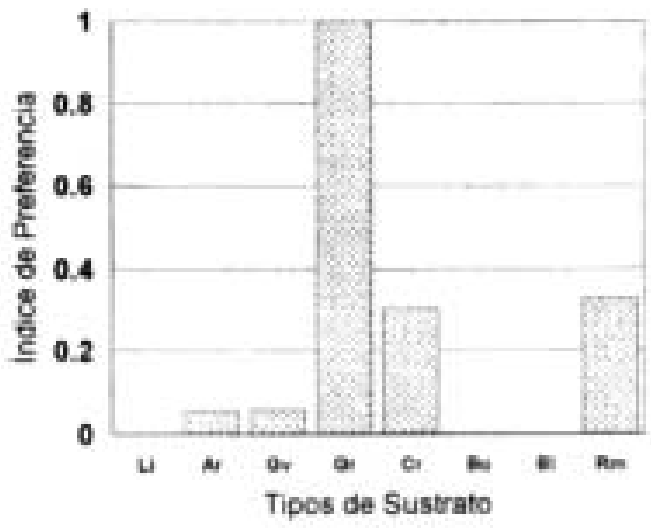

Cacho juvenil

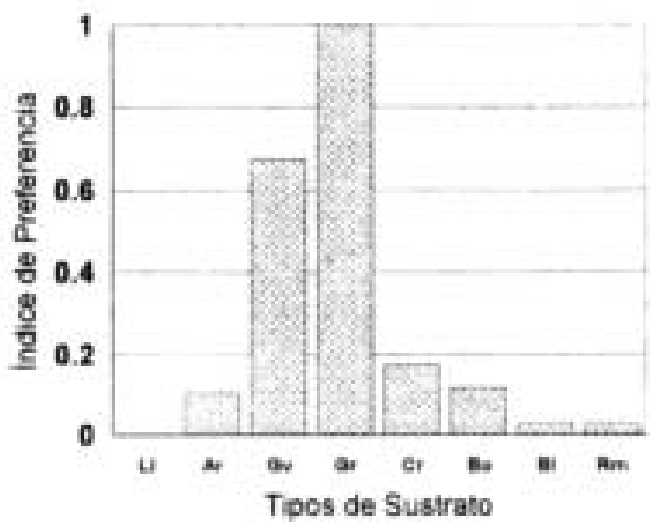

Cacho alevin

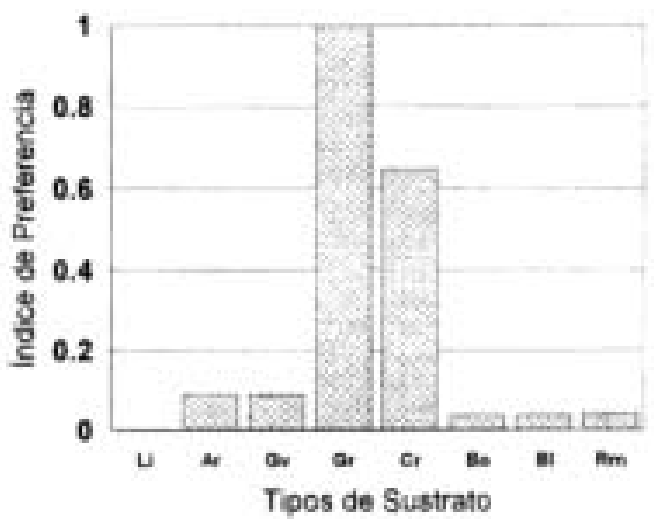

Figura 4. Curvas de preferencia según tipos de sustrato. Li: Limo, Ar: Arena, Gv: Grava, Cr: Cantos rodados, Bo: Bolos, Bl: Bloques, Rm: Roca madre. Suitability curves for substrate types. Li: Silt, Ar: Sand, Gv: Fine gravel, Cr: Cobbles, Bo: Medium and small boulders, Bl: Large and very large boulders. 
menes, cercanos al sustrato y en zonas medianamente profundas $(40-60 \mathrm{~cm})$. Los cachos prefieren sobre todo las gravas, mientras que para los barbos la preferencia es máxima por la arena y decrece al aumentar el diámetro de las partículas. Esto parece responder a sus diferentes estrategias alimenticias, habiéndose observado que los barbos (de todas las edades) suelen extraer su comida de los sustratos menos gruesos, mientras que los cachos se alimentaban casi siempre de los macroinvertebrados que descienden suspendidos en la columna de agua, y cuya abundancia es mayor en las zonas de grava y gravilla. Se anotaron en total 18 barbos y 59 cachos mientras comían (en su mayoría adultos y juveniles), a lo que hay que sumar numerosas observaciones previas y posteriores al muestreo subacuático sensu stricto. En cuanto a los elementos de refugio también los barbos juveniles se encontraban más asociados a ellos respecto a los cachos (47\% de barbos frente a un $15 \%$ de cachos), lo que podría explicarse por su gran diferencia de tallas, al igual que en el caso de los adultos.

Los individuos alevines de las dos especies tienen una preferencia muy similar en cuanto a sustrato (gravas y cantos rodados) y también en cuanto a velocidades focales, si bien el tema del sustrato podría deberse a la gran disponibilidad de estos tipos en el tramo de estudio. Las curvas de preferencia del barbo alevín muestran velocidades (focales y medias) mayores que para los adultos, lo cual explicamos de dos modos. Por una parte el escaso número de individuos adultos y su preferencia por los microhábitats más lentos y profundos, ocupando los alevines microhábitats más diversos y por lo tanto algo más rápidos. Por otro lado, la gran representatividad de las velocidades bajas en el tramo no nos permite afirmar si estas diferencias se deben a cambios debidos al crecimiento, o a la disponibilidad de microhábitats. La preferencia para profundidad es parecida a la de los juveniles en el caso del barbo, mientras que para los cachos es muy parecida a la de los adultos. Como en las demás etapas de desarrollo los barbos aparecieron significativamente más asociados a elementos de refugio, con un $60 \%$ de los individuos frente a un 45
$\%$ de los cachos. Los 15 casos de barbo se refieren a la protección aportada por elementos del sustrato (cornisas y cuevas) mientras que en los cachos alevines también toma mucha importancia la vegetación (62 casos de 107). Consideramos que la velocidad y la capacidad de refugio son los elementos que mejor definen los microhábitats escogidos por los alevines.

En ambas especies, las tres etapas de desarrollo mostraron preferencias de altura focal muy parecidas, encontrándose generalmente cerca del lecho, aunque los alevines del cacho se suelen situar en cardúmenes más distribuidos en la columna de agua. Los valores del "porcentaje de altura focal" no revelaron diferencias claras entre especies ni estados de desarrollo. Grossman \& Sostoa (1994a, b) encontraron que para Barbus graellsii y Leuciscus cephalus los mayores individuos ocupan zonas más profundas y más lejos del sustrato. A pesar de tratarse de distintas especies, pensamos que presentan muchas similitudes, ya que coincidimos en la primera observación en el caso del Barbus bocagei, si bien en el caso del cacho no ha sido claro. En cuanto a la altura focal, el cacho mostró situarse en un rango más amplio de la columna de agua, si bien los máximos de preferencia resultan poco reveladores en cuanto a estas diferencias. Así pues, nuestras observaciones solamente nos dicen que el solapamiento interespecífico es importante en cuanto a esta variable, tal y como afirman Grossman et al. (1987 a, b).

Como el equipo de Grossman \& Sostoa, hemos encontrado que el cacho prefiere velocidades mayores al ir creciendo, y también mayores que el barbo. Esto último parece responder a la ocupación por el cacho de una zona más amplia en la columna de agua y el carácter omnívoro del barbo, que encuentra posiciones energéticamente beneficiosas más cerca del fondo, al poder obtener alimento directamente sobre el sustrato. También coincidimos en que la profundidad es una variable diferenciadora en cuanto a las distintas tallas (aumentando en general con el tamaño del pez), aunque hemos encontrado el caso discrepante del cacho alevín. En general pensamos que la profundidad no 


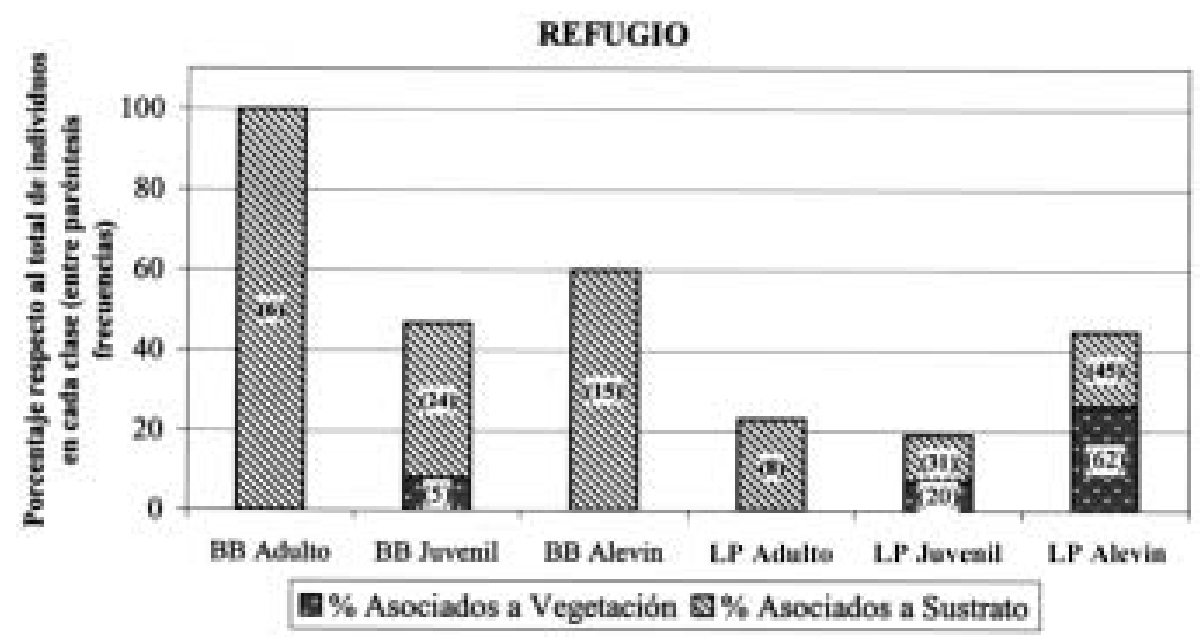

Figura 5. Porcentajes de uso de refugio respecto al total de individuos registrados en cada especie y etapa de desarrollo, así como frecuencias absolutas en cada caso. Percentage use of cover calculated over total number of individuals of each species and life stage. Absolutes frequencies are also shown in each case.

revela diferencias interespecíficas relevantes. Por último, también hemos encontrado que el sustrato ha sido muy poco relevante y no ha reflejado diferencias interespecíficas ni dependientes del estado de desarrollo.

Hemos consultado los resultados de probabilidad de uso (categoría II) obtenidos en Portugal por Costa et al. (1988). Los adultos y juveniles de Barbus steindachneri mostraron una máxima probabilidad para las velocidades más bajas $(<0,1 \mathrm{~m} / \mathrm{s})$, lo que se asemeja mucho a nuestros datos. De igual modo la preferencia de los adultos por las gravas y la roca madre es muy alta, aunque los juveniles prefieren esta última al contrario que los estudiados en el Jarama. Por último las profundidades muestran distribuciones muy distintas.

Al comparar nuestros datos con los de Costa et al. (1988) nos hemos encontrado con varios problemas, además de tratarse de distintas especies. En primer lugar las diferencias metodológicas son muy notables, ya que dicho equipo empleó la técnica de captura con pesca eléctrica. Este método no permiten estimar el punto focal del pez, no es apropiado para la observación de los peces en determinadas zonas del río (rabiones, zonas con espuma, cornisas sumergidas, vegetación acuática, etc.) y no aporta mucha fidelidad a la hora de conocer el comportamiento del pez (Bovee, 1986; Heggenes et al., 1990, 1991). Por estas razones escogimos el método de observación directa bajo el agua, que frecuentemente no se encuentra con los anteriores problemas (Heggenes, 1994). En segundo lugar, no tenemos datos de disponibilidad de hábitat sobre las zonas muestreadas en Portugal, por lo que no podemos comparar las condiciones de la zona de estudio. En tercer lugar, el número escaso de individuos observados en algunos casos, particularmente el barbo adulto, no nos permite extraer conclusiones sólidas de esta comparación.

García de Jalón et al. (1997) realizaron unas curvas de preferencia de categoría I para los ciprínidos reófilos de la Península Ibérica. Estas curvas muestran una preferencia máxima de velocidad a los $0.2 \mathrm{~m} / \mathrm{s}$ y decreciente al aumentar ésta. Los resultados son muy similares a los que hemos obtenido experimentalmente, si observamos en conjunto las seis curvas de velocidad media. Las curvas de profundidad que presentan, con un 
máximo entre 0.7 y $1.1 \mathrm{~m}$, se asimilan a las de los individuos adultos del Jarama, si bien su preferencia hacia zonas menos profundas es mucho menor, inclusive en el caso de alevines y juveniles que hemos observado. Las curvas de sustrato coinciden en las altas preferencias por la grava y en algunos casos por los cantos rodados, si bien la roca madre no recibió tanta valoración como señalan nuestras observaciones para los adultos, influidos por la disponibilidad de este sustrato en las zonas más profundas.

Con las curvas de preferencia realizadas por Pouilly \& Souchon (1994), para barbos mayores de $220 \mathrm{~mm}$, hemos encontrado ciertas semejanzas. En primer lugar se observa que tanto sus curvas de velocidad como de profundidad para Barbus barbus resultaron ser bimodales, mientras que las nuestras no lo son. Nuestra curva de velocidad coincide totalmente con el primer máximo de preferencia que aparece a los $5 \mathrm{~cm} / \mathrm{s}$, si bien el resto de la curva no coincide con el comportamiento que hemos observado en los adultos. En general se observa un comportamiento similar, al aumentar gradualmente la preferencia con la profundidad. El sustrato es más adecuado conforme aumenta el diámetro de las partículas, lo cual hemos confirmado parcialmente al obtener la roca madre el índice de preferencia máximo. Nuestras curvas son unimodales y no se ha observado ninguna dicotomía en el comportamiento diurno de ninguna de las etapas de desarrollo de Barbus bocagei. Si bien se han realizado numerosas observaciones, hay que añadir de nuevo que el número de registros realizados sobre barbo adulto fue muy bajo. Los muestreos se realizaron todos a plena luz del día, en el centro del verano y con un caudal circulante continuo, de modo que no se ha observado una evolución temporal en el comportamiento de ninguna de las etapas de desarrollo del barbo, ni tampoco del cacho.

Por último, también hemos consultado las curvas de Cowx et al. (1994) para Leuciscus cephalus. Las velocidades resultan muy parecidas, con un óptimo de velocidad entre los 5 y los $45 \mathrm{~cm} / \mathrm{s}$ (en Thames/Trent), que es el rango de velocidades escogidas por los individuos de Leuciscus pyrenaicus que hemos observado. Las profundidades solo coincidieron en los valores más altos de nuestra estación pero nuestro rango de datos es menor al suyo por lo que no podemos comparar en valores altos.

Finalmente los resultados obtenidos solo son válidos in situ para nuestra estación, con las características peculiares que hemos detallado al hablar del área de estudio y del hábitat disponible. Actualmente se continua muestreando en otras cuencas de la Península Ibérica, buscando la diversidad de condiciones de nuestros ríos para estudiar la transferibilidad de resultados y para conocer mejor las preferencia globales y el comportamiento de Barbus bocagei y Leuciscus pyrenaicus.

\section{AGRADECIMIENTOS}

En primer lugar agradecemos su ayuda a Ken Bovee por sus correcciones y comentarios, así como a los compañeros del Laboratorio de Hidrobiología. A Esther Naveiro por su ayuda en muestreos de campo y en la redacción de este trabajo. También a Martín Mayo, que nos ha ayudado desde el principio de la investigación y a las aportaciones críticas del referee encargado de la corrección de este artículo.

\section{BIBLIOGRAFÍA}

BOVEE, K. D. 1982. A guide to stream habitat analysis using the instream flow incremental methodology. U.S. Fish and Wildlife Service, Cooperative Instream Flow Group. Instream flow information paper $12.248 \mathrm{pp}$.

BOVEE, K. D. 1986. Development and evaluation of habitat suitability criteria for use in theinstream fow Incremental methodology. U.S. Fish and Wildlife Service, Cooperative Instream Flow Group. Instream Flow Information Paper 21. 235 pp.

BOVEE, K. D., B. L. LAMB, J. M. BARTHOLOW, C. B. STALNAKER, J. TAYLOR, \& J. HENRIKSEN. 1998. Stream habitat analysis using the instream flow incremental methodology. U.S. Geological Survey, Biological Resources Division Information and Technology Report USGS/BRD1998-0004. $131 \mathrm{pp}$. 
BROTONS, P. A. 1988. Condicionamientos hidráulicos sobre la microdistribución en densidad de las poblaciones de macroinvertebrados de bentos. Estudio en un tramo del río Jarama (Madrid). Proyecto fin de carrera. E.T.S. de Ingenieros de Montes. Madrid.

COSTA, M. J., J. M. GOMES, A. BRUXELAS \& M. I. DOMINGOS. 1988. Efeitos previsiveis da construçao da barragem de Alqueva sobre a ictiofauna do rio Guadiana. Revista de Ciencias Agrarias, XI (4): 143-163.

COWX, I. G., C. S. PITTS, K. L. SMITH, P. J. HAYWOOD \& S. W. F. VAN BREUKELEN. 1994. Factors influencing coarse fish populations in lowland rivers. Nat. Rivers Authority R \& D Report 0429/6/N Y. 120 pp.

GARCÍA DE JALÓN, D., B. GUTIERREZ, F. MARTÍNEZ, M. MORILLO, S. BASELGA \& D. BAEZA. 1997. Realización de la metodología de cálculo de aportaciones ambientales y caudales ecológicos mínimos en la cuenca hidrográfica del rio Tajo. Informe técnico CEDEX. Madrid.

GROSSMAN, G. D., A. DE SOSTOA, M. C. FREEMAN \& J. LOBON-CERVIA. 1987 a. Microhabitat use in a Mediterranean riverine fish assemblage: I. Fishes of the lower Matarrana. Oecologia, 73: 490-500.

GROSSMAN, G. D., A. DE SOSTOA, M. C. FREEMAN \& J. LOBON-CERVIA. 1987 b. Microhabitat use in a Mediterranean riverine fish assemblage: II. Fishes of the upper Matarrana. Oecologia 73: 501-512.

GROSSMAN, G. D. \& M. C. FREEMAN. 1987. Microhabitat use in a mediterranean fish assemblage. J. Zool, 212: 151-176.

GROSSMAN, G. D. \& A. DE SOSTOA. 1994 a. Microhabitat use by fishes in the lower rio Matarraña, Spain: 1984-1987. Ecol. Freshwat. Fish., 3: 123-136.

GROSSMAN, G. D. \& A. DE SOSTOA. 1994 b. Microhabitat use by fishes in the upper rio Matarraña, Spain: 1984-1987. Ecol. Freshwat. Fish., 3: 141-152.
HAMPTON, M. \& M. ACEITUNO. 1988. Direct observation techniques for habitat use criteria development on the Trinity River, Trinity County, California. U.S. Fish and Wildlife Service Biological Report, 88 (11): 159-180.

HEGGENES, J. 1994. Physical habitat selection and behaviour by Brown trout (Salmo trutta) and young atlantic salmon (Salmo salar) in spatially and temporally heterogeneous streams: implications for hydraulic modelling. Proceedings of the first International Symposium on Habitat Hydraulics, Trondheim, Norway: 12-30.

HEGGENES, J., A. BRABRAND \& S. J. SALTVEIT. 1990. Comparison of three methods for studies of stream habitat use by young brown trout and atlantic salmon. Trans. Am. Fish. Soc., 119: 101-111.

HEGGENES, J., BRABRAND, A. \& SALTVEIT, S. J. 1991. Microhabitat use by brown trout (Salmo trutta) and atlantic salmon (Salmo salar) in a stream: a comparative study of underwater and river bank observations. J. Fish Biol. 38: 259-266.

JOWETT, I. G. 1990. Factors related to the distribution and abundance of brown and rainbow trout in New Zealand clear-water rivers. N. Z. J. Mar. Fresh. Res. 24: 429-440.

MAYO, M., B. GALLEGO, D. GARCÍA DE JALÓN \& P. A. BROTONS. 1995. Preferencias de hábitat de la trucha común en la época de freza. Río Dulce, Guadalajara. Limnetica, 11 (1): 49-54.

MANLY, B. F. J., L. L. MC DONALD \& D. L. THOMAS. 1993. Resource selection by animals: statistical design and analysis for field studies. London: Chapman and Hall.

POUILLY, M. \& Y. SOUCHON. 1994. Simulation de l'habitat physique du barbeau fluviatile (Barbus barbus, L. 1758): Choix des modèles biologiques et sensibilité de la réponse. Bull. Fr. Pêche Piscic., 334: 213-225.

WESCHE, T. A., C. M. GOERTLER \& W. A. HUBERT. 1987. Modified habitat suitability index model for brown trout in southeastern Wyoming. $N$. Am. J. Fish. Mgmt., 7: 232-237. 